\title{
Blockchain in FinTech: A Mapping Study
}

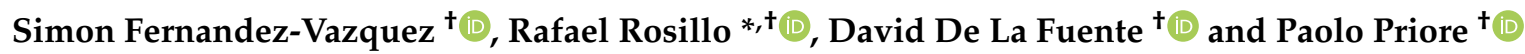

Business Management Department, University of Oviedo, 33003 Asturias, Spain; fernandezvazquez.simon@gmail.com (S.F.-V.); david@uniovi.es (D.D.L.F.); priore@uniovi.es (P.P.)

* Correspondence: rosillo@uniovi.es; Tel.: +34-985-18-26-57

+ These authors contributed equally to this work.

Received: 13 October 2019; Accepted: 6 November 2019; Published: 13 November 2019

\begin{abstract}
Blockchain is currently one of the most important topics in both the academia and industry world, mainly due to the possible effects that the continuing application of this new technology could have. The adoption of this technology by FinTech companies constitutes the next step towards the expansion of blockchain and its sustainability. The paper conducts a mapping study on the research topics, limitations, gaps and future trends of blockchain in FinTech companies. A total of 49 papers from a scientific database (Web of Science Core Collection) have been analyzed. The results show a deep focus in challenges such as security, scalability, legal and regulatory, privacy or latency, with proposed solutions still to be far from being effective. A vast majority of the research is focused into finance and banking sector, obviating other industries that could play a crucial role in the further expansion of blockchain. This study can contribute to researchers as a starting point for their investigation, as well as a source for recommendations on future investigation directions regarding blockchain in the FinTech sector.
\end{abstract}

Keywords: blockchain; FinTech; mapping study; technological challenges; cryptocurrency

\section{Introduction}

Blockchain is one of the most talked-about topics in the business and academic world. It was presented for the first time with Bitcoin in 2008, as a peer-to-peer payment system for electronic transactions which allowed different financial actors to send payments to one another without the intermediation of a central agent (for example a central bank), preventing the double-spending problem [1].

The chain of blocks, or blockchain, is a peer-to-peer network, connected by its nodes that form the chain. Its properties are that of a distributed, transactional database. Once each node in the network verifies the information, it is sent via their public keys to the rest of the nodes [2]. As shown in Figure 1, each block has a unique identification hash that makes reference to its preceding block. Any user with a public or private key can enter the network and have access to the information exchanged in the system network.
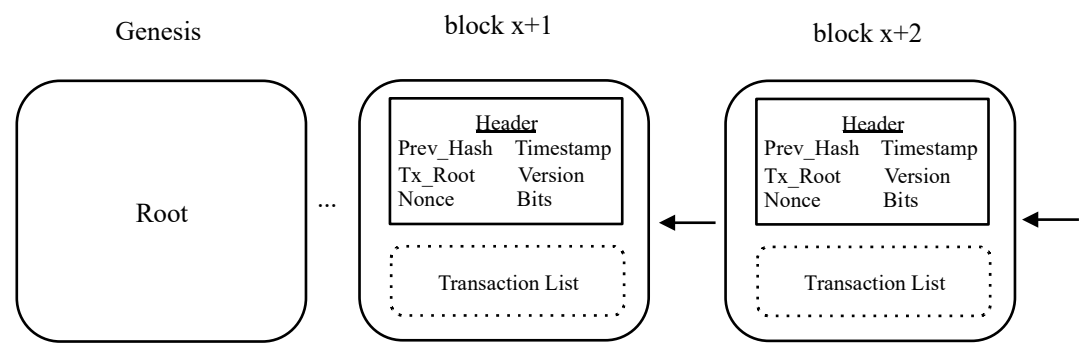

block $\mathrm{x}+3$

Figure 1. Blockchain network. 
Public and private key systems have been developed way before blockchain. In 1976, Diffie and Hellman developed asymmetric cryptography, the first milestone into the development of the key system. In a public key system, two parties are able to send information via a public network, with public techniques and establish a connection that is secure. It works when one party sends the other information enciphered in their respective public keys. In order to decipher the message, the counterparty would use its private deciphering key [3].

The public and private pair of keys are interrelated, meaning that they can only be used in combination. This is obtained through a mathematical algorithm that sets the exclusive relationship between this pair of keys. Public keys can be shared with unlimited parties, whilst private keys must be kept safe and secret [4].

One of the most important players in the blockchain technology are its miners. Miners validate the information in the network by solving cryptographic puzzles and attaining agreement. This procedure makes the chain of blocks secure [5]. Every time one of the miners deciphers the puzzle, a transaction is documented. Due to the reward approach of the blockchain and to incentivize its miners, every time a puzzle is solved Bitcoins are earned. Miners with the greatest resources will be more likely to solve the puzzle first, thus earning the reward. The decentralized environment of Bitcoin is possible due to this structure [6].

When a new miner has access for the first time to a blockchain, it has access to the whole chain, from the genesis to the ultimate validated block [7]. The genesis, also known as the first block or root of the chain, is hard-coded into the client software that supports the valid blockchain. Due to the fact that miners need to solve puzzles, also called proof-of-work (PoW), a new transaction will only be valid once a new block is created and added into the existing blockchain [8]. Blockchain is an asset-agnostic technology. It is capable of storing, record-keeping and transferring all types of assets [9].

This paper introduces a mapping study which aims at comprehending the research topics, limitations, gaps and future trends of blockchain technology in FinTech companies. It can serve researchers as a starting point for their investigation, or as a source of information on future trends regarding blockchain in the FinTech sector. The rest of the paper is organized as follows: Section 2 provides a background on FinTech and smart contracts; Section 3 presents the materials and methods used to conduct this study; Section 4 presents the results of the study; Section 5 discusses the limitations of a systematic mapping study; and Section 6 presents the conclusions and recommendations derived from this study.

\section{Background}

\subsection{FinTech}

Financial technology, also known as 'FinTech', denotes the use of computer programs or other technology to assist the financial industry. The term was used for the first time at the beginning of the 1990s [10] and what started as a word related solely to the financial industry, it soon expanded into other very diverse sectors. Since early 2014, the sector has started attracting the attention of regulators, industry members, customers, and academics [11]. Blockchain in FinTech appeared for the first time as the distributed ledgers of Bitcoin, but has recently attracted consideration from practitioners and researchers [12]. Today, financial institutions and other market participants, mainly due to the development of the blockchain technology, are approving the nature of FinTech and the necessity for research in the academic world given the implications of this technology. Financial innovation is not something new, as it has an extensive history. The development of FinTech throughout history can be divided into three main eras [11].

i. $\quad$ Fintech 1.0 (1866-1967): In this early stage, finance started developing in agricultural states. The use of money, with its main advantage being the transfer of its value, started facilitating financial transactions. Developments in the 19th century of railroads and the invention of 
the telegraph facilitated connections across borders. After the Great War, technology started quickly developing, laying the foundations of the next FinTech era.

ii. Fintech 2.0 (1967-2008): This era is characterized by the rapid expansion of electronic payment systems. In 1968, the Inter-Bank Computer Bureau was founded in the United Kingdom, cementing what today is known as the Bankers' Automated Clearing Services. Regulations in the FinTech world started taking place, mainly due to the collapse in 1974 of Herstatt Bank. The effects of the collapse of the stock market in 1987 (also known as Black Monday), confirmed the suspicion that global markets were technologically linked. Throughout the 1990s, technological advances were made in risk management systems and the development of online consumer banking. The creation of digital banking (back then banks were the sole authorized monetary institutions) attracted more attention by regulators as it created new risks.

iii. Fintech 3.0 (2008-present): The beginning of this era was characterized by the financial turmoil of the years 2007-2008. Trust in the banking system started to be lost, and technological firms started to operate through peer-to-peer networks outside the regulatory framework (in China alone over 2000 platforms were developed). Today, these technological firms and many start-ups are displacing banks at a pace never seen before. Flexible regulations that stimulate entrepreneurship [13] are beginning to be adopted by some countries.

\subsection{Smart Contracts}

The introduction of smart contracts has been key in the development of FinTech. During the last decade, blockchain technology has been constantly evolving. Some of the most relevant products of this evolution are smart contracts. These are not something new, as Nick Szabo introduced the concept in 1994. Smart contracts can be defined as a computerized transaction procedure that performs the terms of a contract. This means that all the contractual clauses are embedded in the computer of the individuals performing the transactions [14]. As these contracts are automatically executed when certain conditions are met (the codes in the algorithm that conform the smart contracts specify these conditions), there is no need for a central authority or third-party support these transactions. As shown in Figure 2, the blockchain is represented on the lower part. Parties involved in the transaction (for example Party A sends units of currency Y to Party B and obtains units of currency Z) are represented in the upper part. Parties exchange this information through their keys (public and private), and consensus of this transaction is reached through mining. The transaction can only be completed with the creation of a new block.

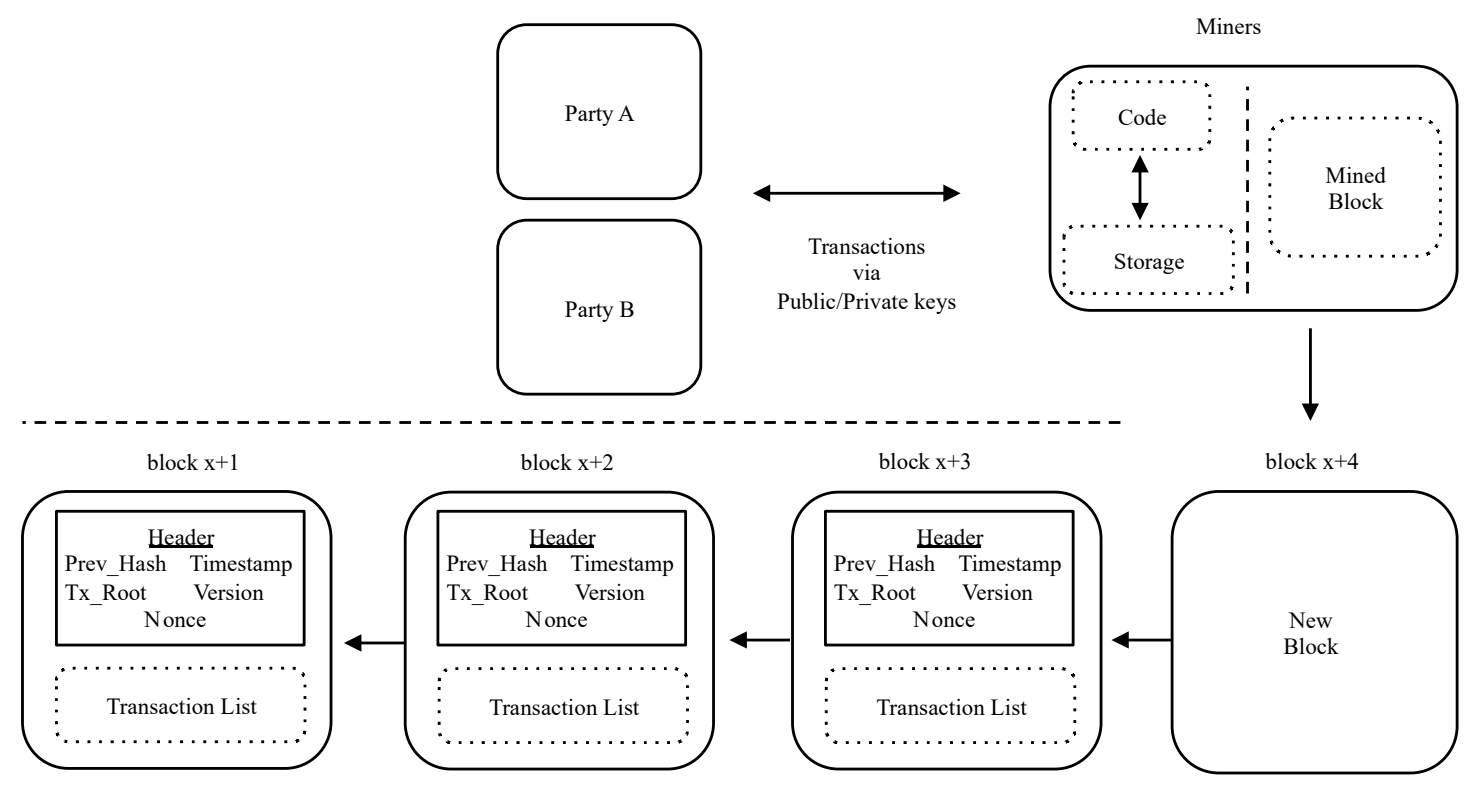

Figure 2. Smart contract system. 
There are many different languages in which smart contracts can be coded, Ethereum being one of the most relevant to date. Ethereum has been proven to be extremely reliable when preventing the double spending problem, although, in order to attain this, a high level of difficulty is added [15]. Currently, the platforms that support blockchain's smart contract are Ethereum and Hyperledger [16].

Ethereum uses its own language, just as any other computer program. It has a consensus procedure that details the way in which the nodes forming the network extend the blockchain. A particularity of Ethereum is that blocks are added based on the strength of the nodes that form the network, through what is called a lottery. This means that nodes with a higher degree of computational strength have more chances of winning this lottery than the ones with less computational strength. Malicious nodes, which could access to win this lottery and add improper contract executions, are automatically removed from the blockchain [17].

\section{Materials and Methods}

A systematic mapping study was the research method used to conduct this study. The objective of a systematic mapping study is to present an outline on an investigated area, establishing and quantifying research evidence on a subject. By performing a mapping study, gaps in the research on a certain topic come to light, which could be considered as weak areas or areas of future study. In order to perform this mapping study, the processes outlined by Petersen [18] and Kitchenham [19] have been followed. The objective of this investigation is to carry out an investigation related to blockchain technology in FinTech. This study provides an indication of the area of research of blockchain technology in FinTech as of today. The Prisma Checklist is provided in Appendix A. Figure 3 shows the systematic mapping study process. It is comprised of five process steps (in the upper part) with their respective five outcomes (in the lower part).

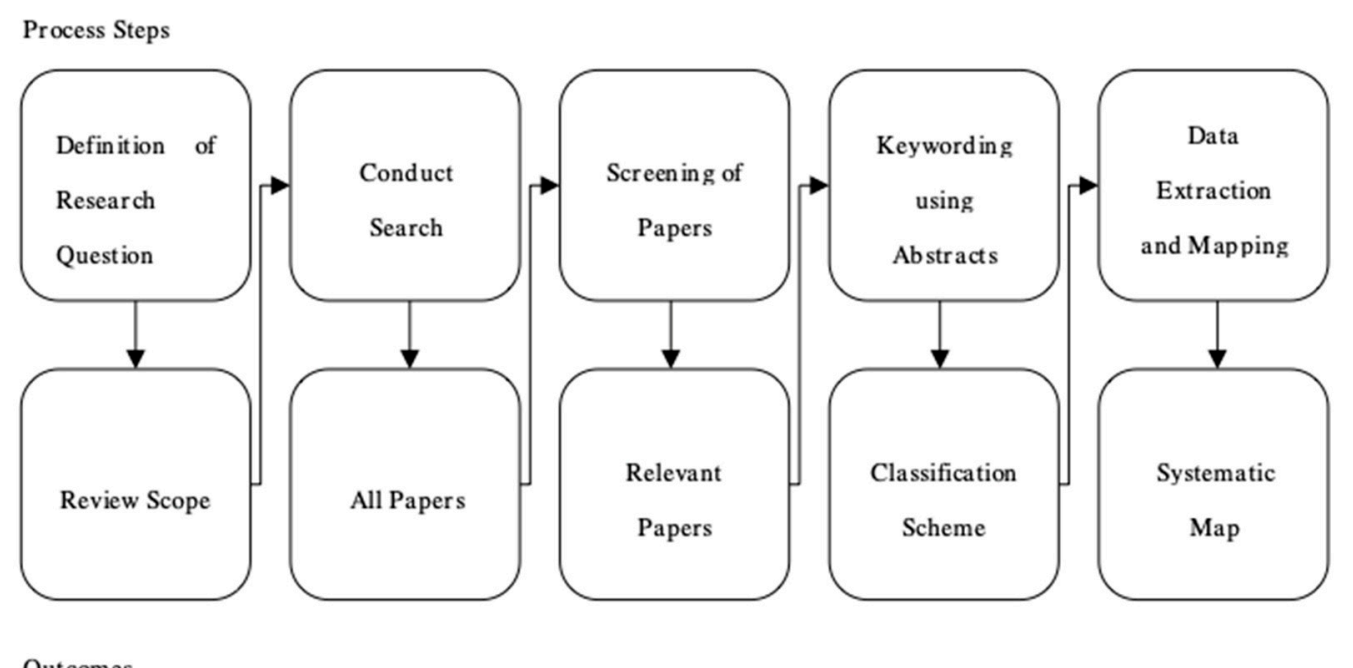

Figure 3. Systematic mapping process.

\subsection{Definition of Research Questions}

A systematic mapping study is characterized by providing an outline or overview of the research scope. It is a crucial link in the chain of evidence that extends from the academic research literature to the practical application, considering that both practitioners and academics use journals as a source of information [20]. A well-executed, scientifically accurate mapping study on a clearly defined topic is invaluable as it outlines current research, defines the limits of what has and has not been investigated and shows the main gaps in the literature research. This analytical angle is extremely important and serves as a highlight of relevant additional issues [21]. This study helps identify the amount and types or research that have been done within the industry. Diverse attributes can be mapped, such as 
publication date trends or the specific topics within the explored area. Research questions (RQs) can help outline these topics more easily.

RQ1: What trends by publication year can be observed?

In order to analyze the trends of blockchain in the academic world, a study of the publication year for the research papers is performed.

RQ2: What types of documents (articles, book chapter, reviews) are being published in blockchain?

Publication channels as a target of research give an idea of the kind of investigation that is being developed within blockchain.

RQ3: What are the main research topics addressed in the current blockchain research?

In order to explore the main topics that have been studied in blockchain, a categorization has to be made. This is one of the most important questions throughout the systematic mapping study carried out, as it serves as a basis to create an overall understanding of the current areas of research. This mapping study will assist researchers to have a wider understanding of this area and serve as a milestone for further investigation.

RQ4: What are the main challenges/limitations in the current blockchain research?

In order to explore the problems and challenges that the development of blockchain in FinTech is experimenting, the main issues will be categorized. By mapping the limitations of the technology, further research can be conducted in a specific area of importance.

RQ5: What are the main gaps in the research in blockchain?

The identification of gaps can help investigators explore areas that have not yet been explored. This will help find answers to questions that have not yet been solved regarding the blockchain technology. Thus, a systematic mapping study provides information on research areas but also on existing research gaps.

RQ6: Where is the blockchain technology moving in the near future?

The above five questions set the groundwork to establish possible future research directions. It is of high importance to address this question in order to lay the groundwork for future research in blockchain and the applications of this technology in the years to come.

\subsection{Conducting the Research}

The second stage after the definition of the research questions is to conduct the search. A search protocol, which will be followed, specifies the approaches that will be taken into account when performing this systematic review. This protocol helps to mitigate the probability of researcher bias. Without this protocol, for instance, it could be probable that the researcher expectations or pre-defined conclusions would drive the selection of individual studies and not the other way around [19]. By this means, an independent third-party (i.e., an independent peer reviewer) could follow this protocol and achieve identical or very similar results.

This search has been performed during the January-March 2019 period. Once the protocol was designed and tested, the search engine/database used was the Web of Science Core Collection (Clarivate Analytics, Philadelphia, USA), which comprises over 1 billion cited reference indexed from journals, books, and proceedings and 21,000 unique global journals covering 254 disciplines. This database contains only high-quality, peer-reviewed papers from different sources.

Within the Web of Science Core Collection, the keyword 'blockchain' was used in the search engine. Since the goal of this mapping study is to map papers related to the blockchain technology in FinTech, no searches for specific terms such as Bitcoin or smart contracts were made. Within the Core Collection, a search was firstly conducted for publications with the word 'FinTech' (or 'financial technology') in the title, abstract or keyword. Also, in order to withdraw subjectivity from the search, there was no manual search involved to retrieve papers or literature that were not contained in the Web of Science Core Collection database (i.e., grey literature such as unpublished papers, Master's or $\mathrm{PhD}$ theses, documents not published as a scientific work, or Google Scholar). 


\subsection{Screening of Relevant Papers for Inclusion or Exclusion}

In order to perform a screening for the relevant papers to be included in the systematic map, the protocol defined by Dyba [22] was adopted. During the first screening phase, papers were screened based on their titles and abstracts. Papers that did not have appropriate titles for the study were excluded. In circumstances where it was not clear whether the title was appropriate, it was passed on to the next phase for further reading. During the second phase, the abstracts of all the papers that passed the first screening phase were read. In this phase, papers with the following characteristics were rejected:

i. Papers where the full text was not available;

ii. Papers written in any other language than English; and

iii. Papers that had other relevance different from blockchain applications in FinTech.

Papers that fulfilled the criteria in both phases passed on to the next stage.

\subsection{Abstract Keywording}

Keywording search is a very useful way to reduce time needed when classifying the results performed and certifying that all studies have been taken into account. In order to perform a keyword search, two steps must be performed. The first one is the reading of the abstract and the identification of key concepts that reveal the contribution of the paper [18]. Once this was completed, a combination of keywords from different papers was performed in order to acquire an understanding of the nature of the search. These keywords were used to form a set of categories in the mapping study. After the categories were shaped, the selected papers were read. Then, if a paper that was initially clustered under a specific category was more relevant under a new category, it was updated accordingly, as it can be seen in Figure 4.

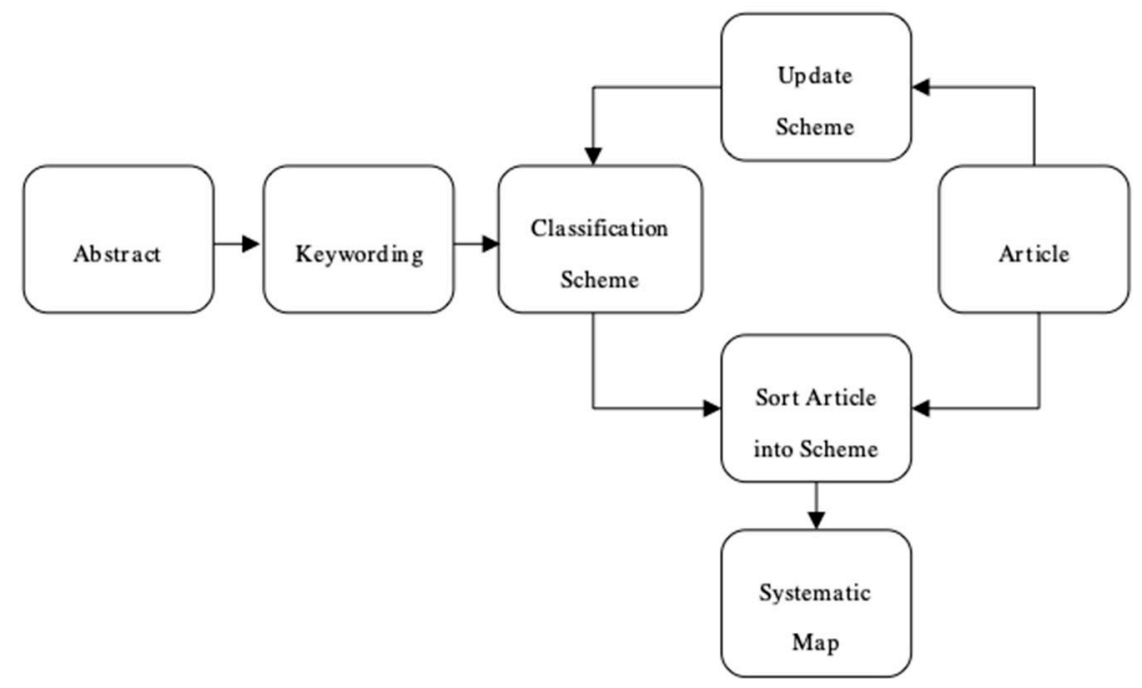

Figure 4. Protocol for building the classification scheme.

\subsection{Data Extraction and Mapping of Papers}

Once the data was obtained from the Web of Science Core Collection database, as previously described, it was sorted in an Excel spreadsheet with the following headers: author(s) name(s), title, source (article, review, book), abstract, and publication year.

\subsection{Paper Information}

The search of results can be seen through the PRISMA diagram in Appendix B. The initial search of the term blockchain in the Web of Science database gave 1,786 results. As it has been previously 
described, the title and abstract keywording led to the selection of 52 papers. The reason for the exclusion of a high number of papers was the absence of correlation between blockchain and Fintech. A vast majority of the papers related to other scientific domains.

After this initial selection of 52 papers, the guidelines for the inclusion of the relevant papers (Section 3.3) were followed. One paper was found to be duplicate, and two papers were eliminated due to the low correlation to the study of blockchain from a FinTech perspective. This process resulted in the final selection of 49 papers. The full list of the selected papers can be seen in Table 1.

Table 1. Publication channels

\begin{tabular}{|c|c|}
\hline Journal & Author(s) \\
\hline IEICE Transactions on Fundamentals of Electronics Communications and Computer Sciences & [23] \\
\hline Electrical Engineering in Japan & [24] \\
\hline Software Engineering and Algorithms in Intelligent Systems & [25] \\
\hline Accounting and Finance & [26] \\
\hline Journal of Economics and Business & [27] \\
\hline Production and Operations Management & [28] \\
\hline Quality-Access to Success & {$[29,30]$} \\
\hline European Business Organization Law Review & [31] \\
\hline NMIMS Management Review & [9] \\
\hline Strategic Change-Briefings in Entrepreneurial Finance & [32] \\
\hline International Journal of Environmental Research and Public Health & [33] \\
\hline Proceedings of The International Conference on Business Excellence & [34] \\
\hline Technological Forecasting and Social Change & [35] \\
\hline 2018 IEEE International Conference on Intelligence and Security Informatics (ISI) & [36] \\
\hline Journal of Investment Management & [37] \\
\hline Blockchain Technology: Platforms, Tools and Use Cases & [38] \\
\hline $\begin{array}{l}\text { Handbook of Blockchain, Digital Finance, and Inclusion, Vol 1: Cryptocurrency, Fintech, Insurtech, } \\
\text { and Regulation }\end{array}$ & [39] \\
\hline 2018 9th IFIP International Conference on New Technologies, Mobility, and Security (NTMS) & [40] \\
\hline Journal of Money Laundering Control & [41] \\
\hline Australian Feminist Studies & [42] \\
\hline Internet Science, INSCI 2017 & [43] \\
\hline Emerging Markets Finance and Trade & [44] \\
\hline Financial and Credit Activity-Problems of Theory and Practice & [45] \\
\hline 2018 6th International Symposium on Digital Forensics and Security (ISDFS) & [46] \\
\hline Journal of Management Information Systems & [47] \\
\hline Journal of Risk Finance & {$[48,49]$} \\
\hline Review of International Business and Strategy & [50] \\
\hline International Journal & [51] \\
\hline Financial Innovation & [52] \\
\hline Business Horizons & [53] \\
\hline Enfoque UTE & [54] \\
\hline Computer & [55] \\
\hline Symmetry-Basel & [56] \\
\hline
\end{tabular}


Table 1. Cont.

\begin{tabular}{|c|c|}
\hline Journal & Author(s) \\
\hline Electronic Commerce Research and Applications & [57] \\
\hline 2017 ITU Kaleidoscope: Challenges for a Data-Driven Society (ITU K) & [58] \\
\hline 2017 IEEE II International Conference on Control in Technical Systems (CTS) & [59] \\
\hline 2017 IEEE 1st International Conference on Cognitive Computing (ICCC 2017) & [60] \\
\hline 2017 AEIT International Annual Conference & [61] \\
\hline Geoforum & [62] \\
\hline $\begin{array}{c}2017 \text { IEEE 13th International Conference on Wireless and Mobile Computing, Networking, and } \\
\text { Communications (WIMOB) }\end{array}$ & {$[63]$} \\
\hline 2017 13th European Dependable Computing Conference (EDCC 2017) & [64] \\
\hline Enterprise Applications, Markets and Services in the Finance Industry, Financecom 2016 & [65] \\
\hline $\begin{array}{c}\text { Proceedings of The 6th International Conference on Computing and Informatics: Embracing } \\
\text { Eco-Friendly Computing }\end{array}$ & [66] \\
\hline 2017 IEEE 13th International Symposium on Autonomous Decentralized Systems (ISADS 2017) & [67] \\
\hline $\begin{array}{c}2017 \text { 19th International Conference on Advanced Communications Technology (ICACT)_Opening } \\
\text { New Era Of Smart Society }\end{array}$ & [68] \\
\hline Recent Developments in Intelligent Systems and Interactive Applications (IISA 2016) & [69] \\
\hline Banking Beyond Banks and Money: A Guide To Banking Services in the 21st Century & [70] \\
\hline
\end{tabular}

\section{Results}

\section{1. $R Q 1$}

\subsubsection{Year of Publication}

Figure 5 shows the year of publication of the selected articles. Due to the fact that FinTech in academia is a rather new subject (blockchain was introduced in 2008), it is not a surprise that only $2.0 \%$ of the selected articles were from the year 2016. The core of the academic publications are found in the years 2017 (38.8\%) and 2018 (53.1\%). Finally, 6.1\% of the selected articles in 2019 (as of march) discussed blockchain in FinTech. If this rate keeps steady, the number of publications in 2018 will be surpassed. This shows that blockchain in FinTech is a very recent research are and that it is quickly expanding. This increase in the publications shows a deep interest in the matter.

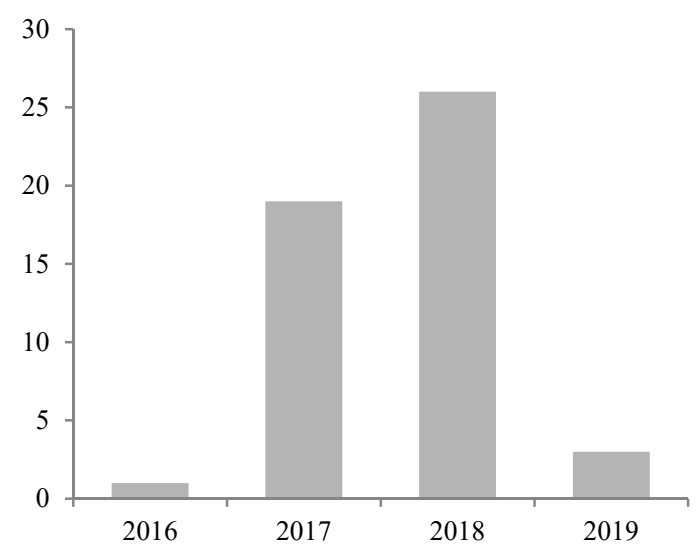

Figure 5. Selected articles published by year. 


\subsubsection{Geographical Distribution}

Regarding the geographical distribution for the selected papers, the main country of publication was the USA with 21 papers (42.9\%), followed by the UK with 11 papers $(22.4 \%)$ and Switzerland with 6 papers $(12.2 \%)$. The rest of the countries had two or less papers published (Figure 6). This distribution shows that, although the publications are mainly concentrated in three countries, there is a high diversification amongst continents that are presently researching blockchain applications in FinTech around the globe.

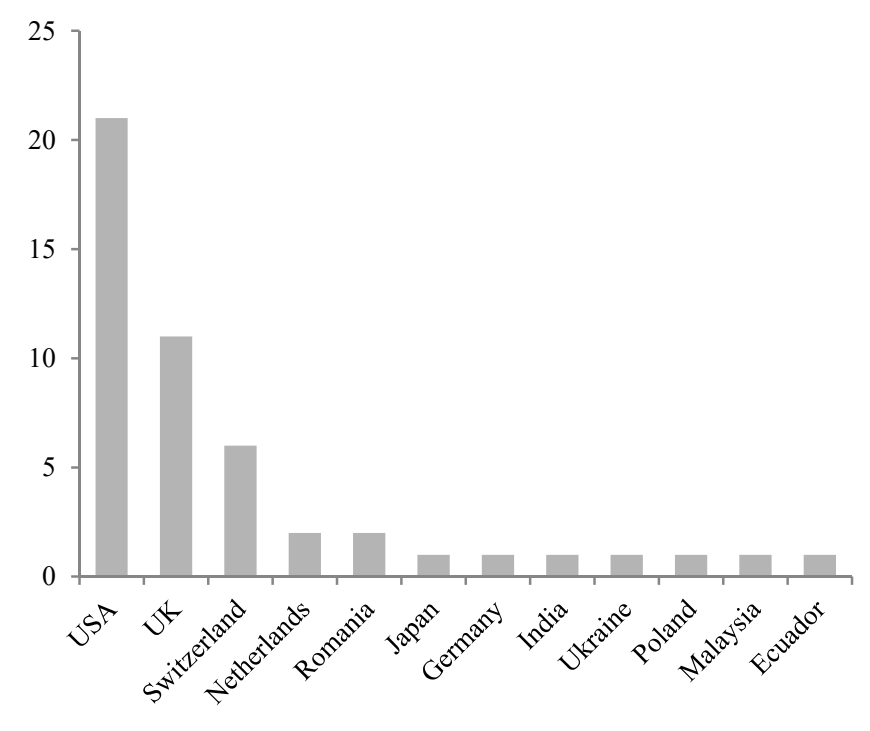

Figure 6. Selected papers geographical distribution.

\section{2. $R Q 2$}

\section{Source of Publication}

Figure 7 shows the publication source for the selected papers. The results from the search show that $55.1 \%$ of the selected papers were articles (27), $34.7 \%$ were proceedings papers (from conferences and symposiums) (17), only $6.1 \%$ of the papers being book chapters (3) and $4.1 \%$ reviews (2).

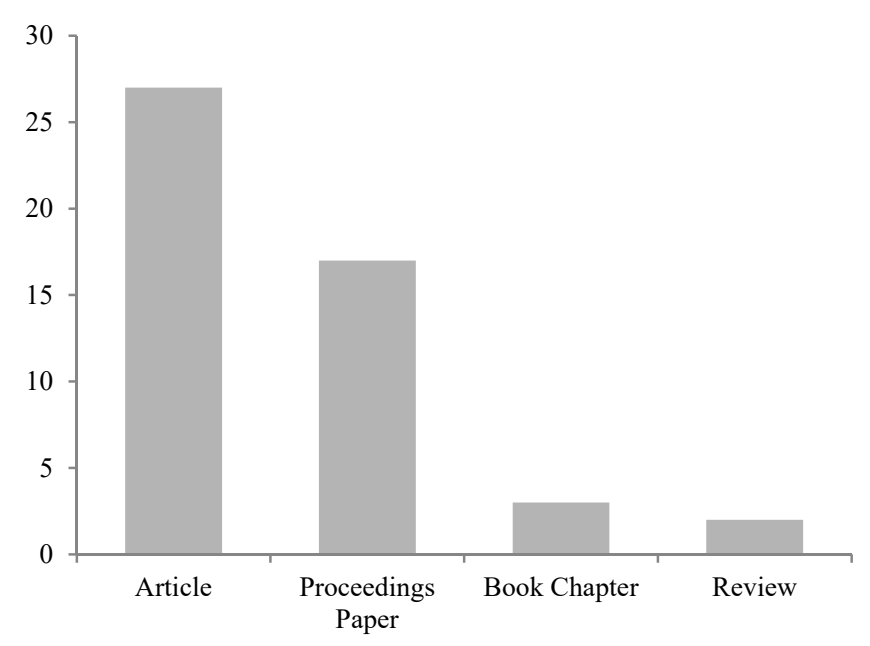

Figure 7. Selected papers source of publication.

Table 1 shows the publication channels for the selected papers. 


\section{3. $R Q 3$}

Summary of the Topics

Figure 8 shows the topics identified through the review of the final selected papers.

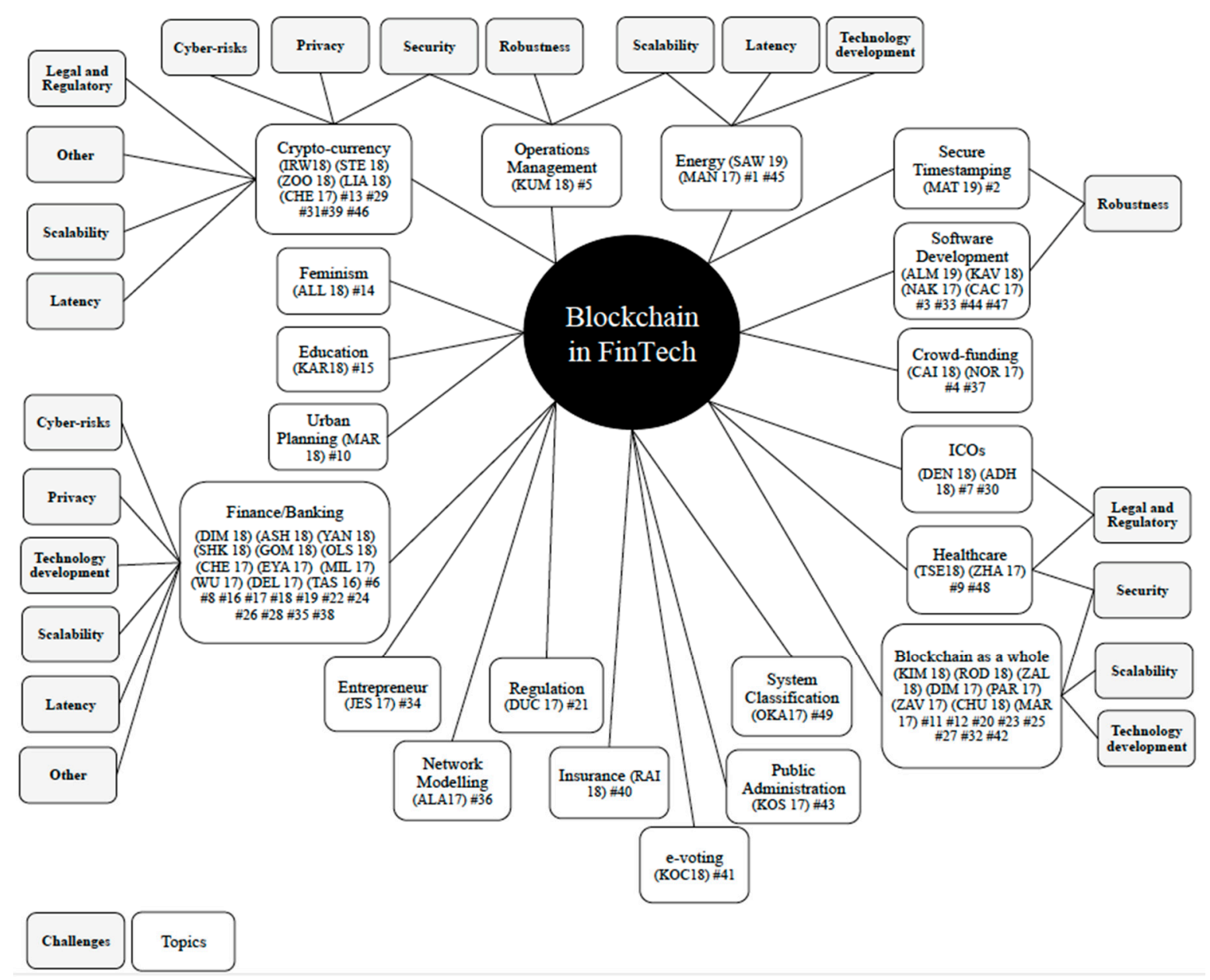

Figure 8. Summary of the main topics and key challenges of the selected papers.

The results of the search performed show that banking and finance are the main topics of the current research in blockchain applied to FinTech, being in $24.5 \%$ the main topic (12 papers). It is not surprising that a wide spectrum of academic research has focused on the banking and finance sector, as blockchain started precisely in these areas. The second most talked-about subject is blockchain and its applications in a wide manner, from the introduction of the technology to the application of the ledger technology to FinTech companies, with 16.3\% (8 papers). The third and fourth topics with the highest amount of interest are cryptocurrencies with $10.2 \%$ (5 papers) and blockchain software development, with $8.2 \%$ (4 papers).

\section{4. $R Q 4$}

\subsubsection{Classification of the Main Challenges for the Selected Papers}

This section presents the classification of the selected papers. Once the papers were read, a classification based on the findings was made. Due to the fact that most of the challenges represented in the papers were related to the classification presented by Treiblmaier [71], this classification was used. The challenges included were: scalability, robustness, latency, legal and regulatory, cyber-risks, security, and privacy. Additionally, a new classification type was identified: technology development. This is a 
very important attribute as blockchain is a rather new technology and presents development challenges. Finally, a classification under the name of 'others' was also used. It comprises all the elements that cannot be classified in the aforementioned categories. The classification is shown in Figure 9.

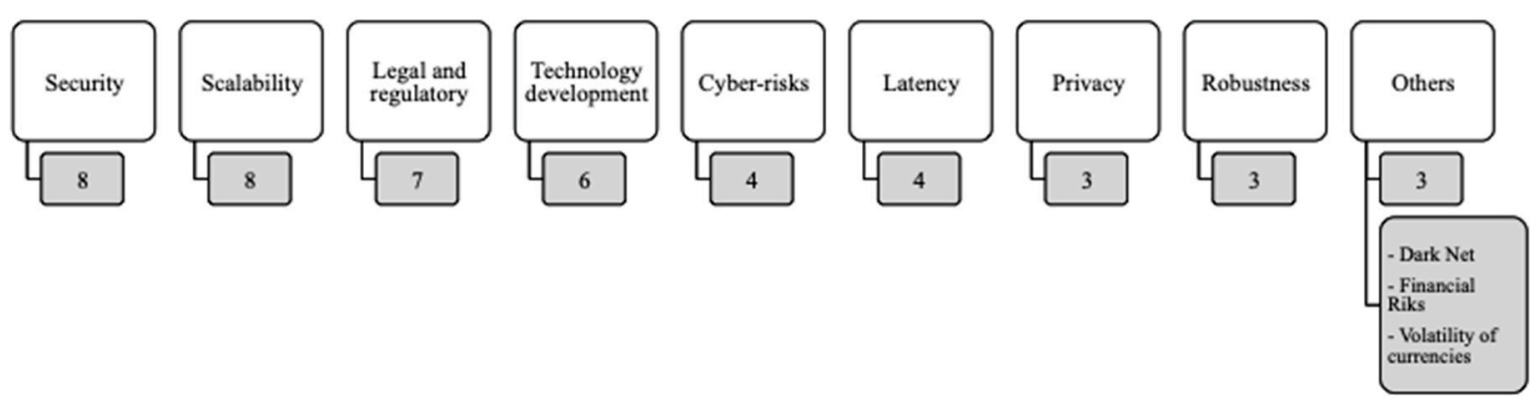

Figure 9. Classification of the main challenges for the selected papers.

\subsubsection{Security}

Security was one of the most talked-about challenges in the selected papers, with eight papers addressing this issue. Different topics inside security were identified, such as $51 \%$ attack, wallet security, cryptographic security, and other issues.

- $51 \%$ attack: One of the most recurrent topics identified related to security has been the $51 \%$ attack to the network. This arises when an attacker has more than $50 \%$ of the mining capability, meaning full control of the blockchain. Whenever there is a fork in the blockchain (two different miners answering the puzzle at the same time), the block that is not selected by the rest of the peers in the network will become worthless. If an attacker has $51 \%$ of the mining capacity in a network, and therefore full control, false transactions can be included in the blockchain. There are five main steps that create the $51 \%$ attack: (i) publication of a mining software with bigger expected value; (ii) creation of a pool with stickiness (Ponzi scheme); (iii) creation of unwanted coalitions; (iv) assault of other pools with cannibalized pools; and (v) an eventual switch to members only [56]. Consensuses of the nodes regarding the transactions are also concepts addressed by other authors [34].

- Wallet security: Regarding Bitcoin, there are numerous security issues that are addressed. Transactions in the Bitcoin world are created using scripts, which are codes in a programming language. An extensively used method that includes a multiple signature process is called 'multisig'. Even though the creation of scripts helps to solve a wide variety of problems, there is a possibility that a transaction is not correctly configured as the complexity of the script rises. When this occurs, the Bitcoin that uses an incorrectly configured script would be discarded, as it would have no use since the unlocking script will not be able to be generated [56]. Humans that run the mining nodes are responsible for choosing which network they want to join, affecting the security of the blockchain. The bigger the amount of nodes in the network, the more secure it will be. The decision to run a specific form of code, or the shifts in the changes in the performance of the systems, have real-world outcomes, i.e. higher expenses on energy and CPU sequences committed to solve proof-of-work encryptions [62].

- Cryptography: Key cryptography is another major topic within security. In the Bitcoin wallet, information such as the personal key of the address used to generate the unlocking script is stored. Having access to this information would mean leading to a potential loss of Bitcoins, as this information plays a crucial part in the system. Consequently, the Bitcoin wallet is one of the central issues when regarding hacking attacks on Bitcoin. One way to tackle this problem is by using multisig for multiple signatures. Because of the particularities of multisig, which only allows a transaction to be executed with multiple signatures, it can be used to configure the signature of the Bitcoin owner in addition to the signature of the online wallet site. In the event of an attack, 
withdrawal can be avoided since the owner's personal key is not kept, even when the online wallet site is being hacked [56]. Security breaches when using cryptographic tools, intrinsic to the concept of blockchain could have a high operational impact. These same breaches could increase costs for users in the network. For instance, arrangements in certain blockchain networks can lead to alterations in the cost allocation amongst users. In a ledger where users contribute through the maintenance and update of the network, some users, by operating certain nodes in an arranged way, could contribute to a cost increase in the network derived from this arrangement [54].

- Other security threats: Some authors point out that operations through blockchain can lead to high overhead traffic [38]. Other authors identify managed monitory services related to cybersecurity. The main idea behind this is that processes are supervised in real-time and attacks are tackled before they can make much damage. Nevertheless, since these monitoring resources are scarce, it is necessary to sacrifice these resources to process those with the higher possibility of being damaged [28]. For other authors, security improvements in the blockchain, especially those between the participants in the logistics supply chain, would result in less cost (i.e., medicines) [33].

\subsubsection{Scalability}

One of the most important challenges concerning blockchain is its scalability. Today, improving the network's scale is not considered itself as a goal, but as a moving target. One of the main objectives of cryptocurrencies is to be able to attain a similar rate of transactions per second as a centralize system does while maintaining the core of its technology unchanged [38].

Some authors point out that the restrictions on a block's data volume limit the amount of transactions performed [24]. Because of its incapability of acting as an exchange mechanism, due mainly to scalability (and speed), cryptocurrencies have been viewed as a source speculative trading [62].

In Bitcoin, another point of view regarding scalability is related to the technical extension of the system. The non-scalable technical extension of the design of Bitcoin does not allow other features or applications to be incorporated. For example, creators that want to incorporate the Bitcoin system to smart contracts, find it extremely complicated. This is how other systems were born, Ethereum being one of the most relevant examples today [63].

In the near future, blockchain can have an issue regarding storage capacity as transactional histories are constantly added onto one another [54]. A solution to this could be the limitation in the access to write information, and only grant permission to a central intermediary, which would diminish consensus needs [9]. Other authors reflect on the necessity of designing a blockchain that can cope with scalability and coming up with a number of computers, which would be necessary to confirm each transaction [28].

Some of the papers that include scalability problems relate it with another common challenge that will be later discussed: latency. In an experiment conducted by Raikwar et al. [40], the parameters when creating a blockchain should be cautiously selected, as they have a direct impact on the network's latency.

\subsubsection{Legal and Regulatory}

Throughout history, there has been a delay between early implementation and regulatory acknowledgment when dealing with important market innovations. Legal compliance and regulation by the authorities in each jurisdiction is a key requirement if blockchain wants to make its way into the financial system. One of the common denominators after any financial crisis is regulatory belief on stable and optimal rules. However, this framework, which relies on stable and predictable rules, could be inadequate in many cases, especially when introducing a disruptive technology such as blockchain in the equation. The proper nature of FinTech, which has diminished barriers to entry, increased access to financial services and challenged the nature of the financial system, needs a regulation that adapts accordingly. Some authors propose regulatory frameworks, whilst others explore the implications that an unregulated system would have. 
Regulators face a series of dilemmas when addressing blockchain law enforcements. On one hand, the risks for policymakers for not incentivizing innovation through the adequate development of an adapted regulation could mean a loss of a country's competitive advantage. Some FinTech start-ups may even end up moving to more favorable jurisdictions in other parts of the globe. On the other hand, an unregulated situation may boost the creation of criminal organizations that would profit from the lack of laws that govern these activities [51]. This deregulation and its consequences is precisely what has been criticized by Stephan C. [34].

Some authors propose data monitoring as a technology driven regulation, which would make financial systems more efficient and effective, protecting the end users' rights. A dynamic and flexible integration with access to the blockchain by the parties involved (i.e., institutions and regulators), would improve real time oversight. This contrasts with the reforms introduced after the financial crisis in 2008 (the Dodd-Frank Wall Street Reform and Consumer Protection Act in the US), at levels that were not attained since the New Deal [44].

The appearance of initial coin offerings (ICOs), a means by which capitalists raise money in the form of cryptocurrencies which can be then bought and sold to obtain services in exchange, is a subject which has attracted the attention of many researchers. Due to the recent widespread increase of cryptocurrencies, ICOs have become very relevant in the blockchain world. Deng et al. [31] suggest that regulation for ICOs in China is necessary, but not to be banned. The authors explore the regulations that have taken place in other countries and jurisdictions-such as the US, Australia, Canada, Singapore, or Hong Kong-where ICOs have been regulated under the securities law. Adhami et al. [27], suggest that ICOs favors innovation and could have substantial significance when funding decentralized groups of developers.

In line with the need for regulation of ICOs, Gomber et al. [47] suggest that it is key for governments and financial institutions to regulate electronic payment systems (i.e., transfer of funds) that are today the leading target of the FinTech revolution. Regulation in other areas, such as in the supply chain of medicines via blockchain when using the Gcoin system, is suggested by Tseng et al. [33].

\subsubsection{Technology Development}

Blockchain technology is far from having reached its maturity, with the technical implications that this may have. Mills et al. [9], for instance, point out that although the blockchain system is regarded with optimism, there is still a lot of room for the development of its applications as the technology is still at an early stage. Although many industry actors are suggesting applications for this technology, the idea is years ahead of the actual technological development.

In order for the distributed ledger technology to be implemented, widespread participation is needed for it to be successful. Every time a new user is included, the whole community benefits. In this case, and because of the characteristics intrinsic to blockchain, early adopters can be penalized from the use of the technology without sufficient participants, which could lead to a withdrawal in the use of blockchain [54].

Rodrigues et al. [38] warn about the possible consequences of investing in blockchain-based start-ups. They point out that investors need to be sure that the technology used to develop the applications is relevant in order to prevent financial losses. The challenges come from developing a system that maintains the blockchain's original properties (transparency and decentralization) without diminishing others (i.e., performance or confidentiality).

Some authors have investigated into the application of the blockchain technology in sectors such as energy and electric power. Through experiments, Sawa, T. [24] demonstrates that the use of this technology remains limited in these areas. Nevertheless, these experiments may help others solve issues relative to energy and provide new solutions that would help companies and customers integrate their needs through blockchain.

Some authors have also addressed waste of resources connected to the early stage of the technology development. One main problem of the proof-of-work system is that it serves no other purpose than 
assuring the security inside the network. A higher computational power is needed when mining takes place, which results in high-energy expenditure. Some studies suggest that by the end of 2020, the energy consumption in assuring the blockchain's PoW will be equivalent to that of Denmark. Some recent attempts to reduce the energy consumed by the PoW (through the recycling of energy) are far from solving this issue [55].

Today, some development models (Standards Development Organizations and National Standards Organizations) are only open to users who have paid a subscription. This limits the number of users that can make developments into a specific area. In order to overcome this situation, Marsal-Llacuna et al. present a blockchain model for drafting with a token to administer open to sharing [35].

\subsubsection{Cyber-Risks}

Blockchain's permissionless public systems can be used for unlawful purposes. This is due to the fact that the system enables (occasionally completely anonymous) transactions across the globe without the intervention of a central authority. The particularities of the permissionless public systems, which cannot impose rules or obligations to its users in order to verify their identity, foster the development of illegal activities [51].

In recent years, cryptocurrencies have gathered attention for being linked to the drug business, money laundering, and financing of terrorism. These new currencies allow users to purchase any kind of malicious items using, for example, Bitcoins. Ransomware attacks have also taken place, such as WannaCry, which affected more than 300,000 computers in 150 countries. Users with infected computers were asked to pay the sum of $\$ 300$ in Bitcoins to be able to have access to their computers. Investigating the traces of these attacks is a very complex and difficult assignment. Also, users in the blockchain can undergo financial losses if their cryptographic keys or identifications are lost or stolen. These losses are in most cases instant and irreversible without recourse [9].

Irwin et al. [41] point out that a solution could come from policies that would support data sharing between different members from the law enforcement, intelligence entities, and organizations dealing with cyber-security as well as the FinTech industry. The result would be the creation of behavioral models and threat landscapes that could help recognize the identities behind the attacks. Other authors point out for the need for a regulation in order to prevent fraud and money laundering activities [34].

\subsubsection{Latency}

Today, it takes around 10 minutes to create a new block using PoW. Roughly, seven transactions are processed per second, which seems very far away compared to the performance of credit cards, which perform around 2,500 transactions per second on average, peaking at 4,000 and even reaching 45,000 at its maximum (SAW 19) with only a fraction of the electrical power used by Bitcoin. The reasons for this latency are mainly due to cryptographic verification and blockchain's consensus algorithms, which delay the amount of transfers that some systems need to function correctly [54].

Nowadays, Bitcoin block puzzles are solved at a minimum time of three seconds and a maximum of more than 50 minutes. Decreasing the complexity of PoW would diminish the time spent, but could lead up to blockchain forks if the block generation timeframe is very narrow [52]. Zook et al. [62] point out that one of the solutions that some cryptocurrency exchanges and miners are coming up with is to charge higher fees in order to process the transactions quicker, and even include charges to cover risks that embrace slow exchange.

\subsubsection{Privacy}

One of the most important challenges of blockchain is privacy. Blockchain's transparency, which is one of the main assets of this technology, could turn to be a major issue. For instance, two companies which have transactions with one another, could be reluctant to have this information tracked by a third party, due to the commercial and client privacy implications that this may have [55]. Holders of Bitcoin can be tracked by analyzing the transactions they perform, mainly through the use of the public 
keys related to their payments. After the identification of a certain subject, a software tool can be used to create a behavioral map based on the gathered information to have an idea of where that person shopped, the amount spent and the frequency of the transactions. Even external third parties can have access to and analyze this information.

Solutions to privacy issues are proposed by Del Rio, C. [54], where the author reflects on a notary based blockchain system, in which no node in the system has the complete set of information, could allow higher privacy as a trusted third party would assist in validating the transactions. The downturn is that if the information in one or more nodes was fraudulent, the system would collapse as nobody would have a full copy of the ledger and verification would not be possible. Bitcoin Fog or Dark Wallet, which foster anonymity through a series of scripts, could be used as an alternative [41].

\subsubsection{Robustness}

When, after the aforementioned challenges, the blockchain network will display similar characteristics to traditional economic networks, it will mean that the system has achieved its robustness. Nevertheless, today, this technology confronts problems that need to be addressed. Robustness looks precisely into higher availability (meaning no downtime) and a 24/7 service available for transactions.

Some authors, like Matsuura [47], point out that many FinTech and emerging blockchain-based applications lack of a solid service quality. Research is made towards the stabilization of the applications with the help of token valuation interpretation function. The main challenge is to comprehend the new necessities regarding blockchain's software development products that startups need in order to develop their business. Improvement in the software's capacity for decision-making, allowing a higher degree of automation, and even evolving traditional software engineering concepts to make them adaptable to blockchain, are the key factors pointed out by Almeida et al. [25]. In line with this, the optimization of smart contracts in the blockchain would certainly help the network achieve its robustness [28].

\subsubsection{Others}

Other topics that have been found relevant when researching blockchain in FinTech are the dark net, financial risks, and cryptocurrency volatility. We have previously analyzed some of the risks associated with cyber-security, and talked about the problem of users being able to buy illicit items with cryptocurrencies. However, the darknet goes beyond common users, as it has been estimated that only one out of 3,000 web pages are visible to everyday search engines, and between $80 \%$ to $98 \%$ of the information on the Internet resides in the darknet. The degree of security of this net, accessible using, for example, a Tor Browser, is extremely high, and recurrent changes in the codes and the use of techniques of indexing of search engines create a place for any kind of illegal activity [41].

In the blockchain, and when dealing with transactions between financial institutions, another challenge that comes up is how the autonomous system would manage financial risks, such as credit or liquidity risks. How the system handles these risks will have a direct impact on the counterpart's liquidity needs [9]. In line with this, financial players also need to address the handling of variables such as the volatility of cryptocurrencies, which has experience in the last two years huge growth accompanied with violent volatility [34]. The sophistication of the designed scripts in the network is key when tackling these issues and will contribute to the expansion of blockchain in FinTech.

\subsection{RQ5: What Are the Main Gaps in the Research in Blockchain?}

By doing this mapping study, some major research gaps were identified. First of all, most of the research is being done in sectors related to banking and finance. There is very little current research in the application of blockchain for FinTech companies that do not relate to these sectors. For instance, investigation of blockchain in sectors such as durable and non-durable manufacturing, retail and wholesale trade, real estate, construction or utilities, is necessary as they represent the biggest industries 
in the economy. Also, a more detailed focus on how blockchain technology could help FinTech's supply chain is needed.

A second major gap is the only focus on Bitcoin when exploring cryptocurrencies and its uses. There are numerous other currencies, for instance, Ethereum, which has the importance of being used in smart contracts. Another research gap can be found in these types of contracts, since they serve as the basis for most of the transactions that will take place in the future of blockchain. If correctly programmed, smart contracts will be able to execute thousands of transactions in an autonomous way. Throughout the selected papers, there were no specific proposals of application development from a smart contract perspective.

The third research gap was the absence of a technical literature. There were no mathematical models for applications related to FinTech. Papers were mostly descriptive and lacked a technical approach in the sense of developing real tools that would help the major challenges brought into light to be overcome. A more thorough investigation is needed to be able to come up with solutions that would help companies in the FinTech sector that want to start using blockchain technology.

\subsection{RQ6: Where is Blockchain Technology Moving in the Near Future?}

It is not clear where the future research of blockchain in the FinTech is going. Cryptocurrencies such as Bitcoin have attracted interest in recent years, and platforms that trade these currencies are growing in users every day. It is very probable that Bitcoin will continue to be one of the most researched topics in the near future, from both business and technical perspectives. As we have pointed out in the current research gaps, other currencies, such as Ethereum, will probably be of interest as well, largely due to the application in smart contracts. Nevertheless, it seems that Bitcoin has taken the lead in the research and we will have to wait for the irruption of other competitors in academic papers.

As more and more users in the real world start to make use of this technology, researchers will start drawing their attention to these uses and applications. An increase in users means that challenges, such as scalability or security, will have greater importance, and the development of applications that tackle these and other issues will be subject of literature review. Even though blockchain has not reached its maturity, we can observe a growth in the interest that will presumably continue until this technology reaches its full capacity.

\section{Discussion}

\subsection{Limitations of a Systematic Mapping Study}

The most common limitations of systematic mapping studies are publication and selection bias, inaccuracy in data collection and misclassification [72]. Publication bias refers to the issue that negative results are less likely to be published, because it takes longer or are less cited, than positive results. This issue was addressed by the use of the Web of Science Core Collection, which only contains high-quality, peer-reviewed papers. Grey literature (i.e., unpublished papers, Master's or PhD theses, documents not published as a scientific work, or Google Scholar) was not considered as part of the search. This could, to an extent, affect the validity of the findings. However, using a reputable database ensures that the results of the study are of high quality.

Selection bias refers to the misrepresentation of a statistical analysis owing to the criteria used to select publications. This issue was addressed by a careful design of a search protocol. Inclusion and exclusion criteria were rigorously applied, and each selected paper answered the research questions. Nevertheless, there is a possibility that papers that addresses the applications of blockchain in the FinTech sector have been left out if they made little reference to the subject, and the terms FinTech (or financial technology) was not included in the title, abstract or keyword. Based on pilot searches carried out prior to the final search, we believe that the majority of relevant papers discussing the subject have been included. Inaccuracy in data selection and misclassification refer to the possibility of a study's information being extracted in different ways by different reviewers. This issue was addressed by 
asking three authors in the paper to extract and classify the results obtained. When discrepancies arose, discussions were solved by consensus. This resulted in the final 49 papers that were selected.

Additionally, according to Centobelli [73] this mapping study is conducted using manual screening and is based on the researcher's analysis and interpretation of the literature. Their critical perspectives unavoidably influence this process. This fact represents, without a doubt, the main strengths and limitations of literature reviews. As a matter of fact, a certain degree of subjectivity is an essential requirement to develop a mapping study and provide thorough understanding of qualitative aspects.

\subsection{Future Research}

The findings of this paper may be useful for future research. The gaps that have emerged from this mapping study highlight that, although in recent years there is an increasing number of articles published on blockchain, the knowledge framework in the discipline is still scattered and a vast majority of subjects and research directions are still substantially unexplored. Nonetheless, the principal outcomes of the mapping study show that interest in the topic is mounting and its framework is in expansion. Since this mapping study provides a conceptual framework, it sets the grounds to future empirical research and improves the awareness in the field of blockchain in the FinTech sector. Recommendations on the research directions of this technology would comprise:

- Further investigation from a technical perspective to attain solutions to the main challenges and limitations that have been identified. If the issues are tackled in the blockchain network as a whole, FinTech companies will soon be able to adapt and take onboard these solutions to today's problems. The recent interest in the technology (mainly since 2016) comprises many papers dedicated to the explanation of blockchain in a broad manner, but there is a lack investigations tackling its limitations with real solutions.

- Further research on issues such as privacy or latency. These are key issues that have not attracted as much interest as security or scalability but that are fundamental to the development of blockchain technology.

- Further development of smart contracts. If FinTech companies want to succeed in the adoption of blockchain technology, smart contracts are the key to further unleashing its potential.

- Development of applications for FinTech companies outside the banking sector. Other industries such as manufacturing, trade, real estate, construction, or utilities have the capacity to take blockchain technology onboard and apply it for many uses. The introduction of blockchain in the supply chain of these industries could foster even more the attention and interest towards blockchain and help further investigate solutions to its main challenges.

\section{Conclusions}

Blockchain is a decentralized network environment with a shared ledger, in which all transactions are publicly available to its users. Throughout a set of protocols and cryptographic techniques, it provides privacy, security, transparency, and anonymity. Nevertheless, these benefits also set up a list of challenges and limitations that need to be explored. In order to comprehend the academic literature available on blockchain, a systematic mapping study was carried out. The objective of this mapping study was to scrutinize the current status, topics and challenges and limitation of blockchain technology in FinTech companies. A total of 49 papers from the Web of Science Core Collection database were examined. The results show a deep focus in challenges such as security, scalability, legal and regulatory, privacy, latency, cyber-risks, or technology development. Although these issues are identified, the proposed solutions are still far from being effective.

In relation to the specified research questions, the present mapping study aims to pinpoint the main research areas of blockchain in the FinTech sector, the main publication trends and the main gaps in academic literature. This mapping study also offers an extensive understanding of blockchain in the financial technology sector. It highlights that companies willing to adopt this technology should deal 
with upcoming challenges and shows the importance of the efforts in understanding and tackling the aforementioned issues. Although blockchain is currently far from being the solution to problems in numerous sectors, its characteristics set hopes for a greater importance in forthcoming years.

Author Contributions: Conceptualization, S.F.-V., R.R., D.D.L.F., and P.P.; Methodology, S.F.-V., R.R., D.D.L.F., and P.P.; Formal analysis, S.F.-V., R.R., D.D.L.F., and P.P.; Investigation, S.F.-V., R.R., D.D.L.F., and P.P.; Resources, S.F.-V., R.R., D.D.L.F., and P.P.; Data curation, S.F.-V., R.R., D.D.L.F., and P.P.; Writing-original draft preparation, S.F.-V., R.R., D.D.L.F., and P.P.; Writing—review and editing, S.F.-V., R.R., D.D.L.F., and P.P.; Supervision, S.F.-V., R.R., D.D.L.F., and P.P.; Project administration, S.F.-V., R.R., D.D.L.F., and P.P.; Validation, S.F.-V., R.R., D.D.L.F., and P.P.; The authors contributed equally to this work.

Funding: This research received no external funding.

Conflicts of Interest: The authors declare no conflict of interest.

\section{Appendix A}

Table A1. PRISMA checklist.

\begin{tabular}{|c|c|c|c|}
\hline Section/Topic & $\#$ & Checklist Item & $\begin{array}{c}\text { Reported on } \\
\text { Page No. }\end{array}$ \\
\hline \multicolumn{4}{|c|}{ TITLE } \\
\hline Title & 1 & $\begin{array}{l}\text { Identify the report as a systematic review, meta-analysis, or both. } \\
\text { ABSTRACT }\end{array}$ & 1 \\
\hline $\begin{array}{l}\text { Structured } \\
\text { summary }\end{array}$ & 2 & $\begin{array}{l}\text { Provide a structured summary including, as applicable: } \\
\text { background; objectives; data sources; study eligibility criteria, } \\
\text { participants, and interventions; study appraisal and synthesis } \\
\text { methods; results; limitations; conclusions and implications of key } \\
\text { findings; systematic review registration number. } \\
\text { INTRODUCTION }\end{array}$ & 1 \\
\hline Rationale & 3 & $\begin{array}{l}\text { Describe the rationale for the review in the context of what is } \\
\text { already known. }\end{array}$ & $1-4$ \\
\hline Objectives & 4 & $\begin{array}{l}\text { Provide an explicit statement of questions being addressed with } \\
\text { reference to participants, interventions, comparisons, outcomes, } \\
\text { and study design (PICOS). }\end{array}$ & $4-8$ \\
\hline $\begin{array}{l}\text { Protocol and } \\
\text { registration }\end{array}$ & 5 & $\begin{array}{l}\text { METHODS } \\
\text { Indicate if a review protocol exists, if and where it can be accessed } \\
\text { (e.g., web address), and, if available, provide registration } \\
\text { information including registration number. }\end{array}$ & $4-8$ \\
\hline Eligibility criteria & 6 & $\begin{array}{l}\text { Specify study characteristics (e.g., PICOS, length of follow-up) } \\
\text { and report characteristics (e.g., years considered, language, } \\
\text { publication status) used as criteria for eligibility, giving rationale. }\end{array}$ & $4-8$ \\
\hline $\begin{array}{l}\text { Information } \\
\text { sources }\end{array}$ & 7 & $\begin{array}{l}\text { Describe all information sources (e.g., databases with dates of } \\
\text { coverage, contact with study authors to identify additional } \\
\text { studies) in the search and date last searched. }\end{array}$ & $4-8$ \\
\hline Search & 8 & $\begin{array}{l}\text { Present full electronic search strategy for at least one database, } \\
\text { including any limits used, such that it could be repeated. }\end{array}$ & $4-8$ \\
\hline Study selection & 9 & $\begin{array}{l}\text { State the process for selecting studies (i.e., screening, eligibility, } \\
\text { included in systematic review, and, if applicable, included in the } \\
\text { meta-analysis). }\end{array}$ & $4-8$ \\
\hline $\begin{array}{l}\text { Data collection } \\
\text { process }\end{array}$ & 10 & $\begin{array}{l}\text { Describe method of data extraction from reports (e.g., piloted } \\
\text { forms, independently, in duplicate) and any processes for } \\
\text { obtaining and confirming data from investigators. }\end{array}$ & $4-8$ \\
\hline Data items & 11 & $\begin{array}{l}\text { List and define all variables for which data were sought (e.g., } \\
\text { PICOS, funding sources) and any assumptions and simplifications } \\
\text { made. }\end{array}$ & $4-8$ \\
\hline $\begin{array}{l}\text { Risk of bias in } \\
\text { individual } \\
\text { studies }\end{array}$ & 12 & $\begin{array}{c}\text { Describe methods used for assessing risk of bias of individual } \\
\text { studies (including specification of whether this was done at the } \\
\text { study or outcome level), and how this information is to be used in } \\
\text { any data synthesis. }\end{array}$ & $4-8$ \\
\hline
\end{tabular}


Table A1. Cont.

\begin{tabular}{|c|c|c|c|}
\hline Section/Topic & $\#$ & Checklist Item & $\begin{array}{c}\text { Reported on } \\
\text { Page No. }\end{array}$ \\
\hline $\begin{array}{l}\text { Summary } \\
\text { measures }\end{array}$ & 13 & $\begin{array}{l}\text { State the principal summary measures (e.g., risk ratio, difference } \\
\text { in means). }\end{array}$ & - \\
\hline $\begin{array}{l}\text { Synthesis of } \\
\text { results }\end{array}$ & 14 & $\begin{array}{l}\text { Describe the methods of handling data and combining results of } \\
\left.\text { studies, if done, including measures of consistency (e.g., } \mathrm{I}^{2}\right) \text { for } \\
\text { each meta-analysis. }\end{array}$ & $4-8$ \\
\hline $\begin{array}{l}\text { Risk of bias } \\
\text { across studies }\end{array}$ & 15 & $\begin{array}{c}\text { Specify any assessment of risk of bias that may affect the } \\
\text { cumulative evidence (e.g., publication bias, selective reporting } \\
\text { within studies). }\end{array}$ & 18 \\
\hline $\begin{array}{l}\text { Additional } \\
\text { analyses }\end{array}$ & 16 & $\begin{array}{c}\text { Describe methods of additional analyses (e.g., sensitivity or } \\
\text { subgroup analyses, meta-regression), if done, indicating which } \\
\text { were pre-specified. }\end{array}$ & - \\
\hline Study selection & 17 & $\begin{array}{l}\text { RESULTS } \\
\text { Give numbers of studies screened, assessed for eligibility, and } \\
\text { included in the review, with reasons for exclusions at each stage, } \\
\text { ideally with a flow diagram. }\end{array}$ & $6-8$ \\
\hline $\begin{array}{c}\text { Study } \\
\text { characteristics }\end{array}$ & 18 & $\begin{array}{l}\text { For each study, present characteristics for which data were } \\
\text { extracted (e.g., study size, PICOS, follow-up period) and provide } \\
\text { the citations. }\end{array}$ & $6-8$ \\
\hline $\begin{array}{l}\text { Risk of bias } \\
\text { within studies }\end{array}$ & 19 & $\begin{array}{c}\text { Present data on risk of bias of each study and, if available, any } \\
\text { outcome level assessment (see item 12). }\end{array}$ & 18 \\
\hline $\begin{array}{l}\text { Results of } \\
\text { individual } \\
\text { studies }\end{array}$ & 20 & $\begin{array}{l}\text { For all outcomes considered (benefits or harms), present, for each } \\
\text { study: (a) simple summary data for each intervention group (b) } \\
\text { effect estimates and confidence intervals, ideally with a forest plot. }\end{array}$ & $4-18$ \\
\hline $\begin{array}{l}\text { Synthesis of } \\
\text { results }\end{array}$ & 21 & $\begin{array}{l}\text { Present results of each meta-analysis done, including confidence } \\
\text { intervals and measures of consistency. }\end{array}$ & $4-18$ \\
\hline $\begin{array}{l}\text { Risk of bias } \\
\text { across studies }\end{array}$ & 22 & $\begin{array}{l}\text { Present results of any assessment of risk of bias across studies (see } \\
\text { Item 15). }\end{array}$ & 18 \\
\hline $\begin{array}{c}\text { Additional } \\
\text { analysis }\end{array}$ & 23 & $\begin{array}{l}\text { Give results of additional analyses, if done (e.g., sensitivity or } \\
\text { subgroup analyses, meta-regression (see item 16)). } \\
\text { DISCUSSION }\end{array}$ & - \\
\hline $\begin{array}{c}\text { Summary of } \\
\text { evidence }\end{array}$ & 24 & $\begin{array}{l}\text { Summarize the main findings including the strength of evidence } \\
\text { for each main outcome; consider their relevance to key groups } \\
\text { (e.g., healthcare providers, users, and policy makers). }\end{array}$ & $18-19$ \\
\hline Limitations & 25 & $\begin{array}{c}\text { Discuss limitations at study and outcome level (e.g., risk of bias), } \\
\text { and at review-level (e.g., incomplete retrieval of identified } \\
\text { research, reporting bias). }\end{array}$ & 18 \\
\hline Conclusions & 26 & $\begin{array}{l}\text { Provide a general interpretation of the results in the context of } \\
\text { other evidence, and implications for future research. } \\
\text { FUNDING }\end{array}$ & $18-19$ \\
\hline Funding & 27 & $\begin{array}{l}\text { Describe sources of funding for the systematic review and other } \\
\text { support (e.g., supply of data); role of funders for the systematic } \\
\text { review. }\end{array}$ & - \\
\hline
\end{tabular}




\section{Appendix B}

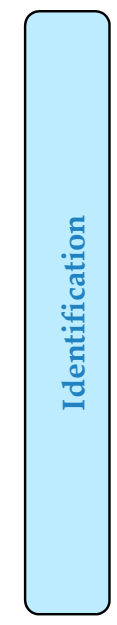

$$
\begin{aligned}
& \text { Records identified through database } \\
& \text { searching } \\
& (\mathrm{n}=1,786)
\end{aligned}
$$

Additional records identified through other sources

$$
(\mathrm{n}=0)
$$
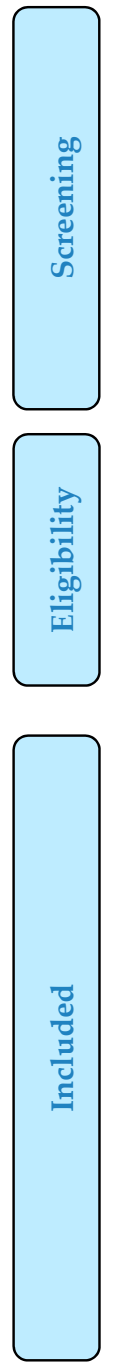

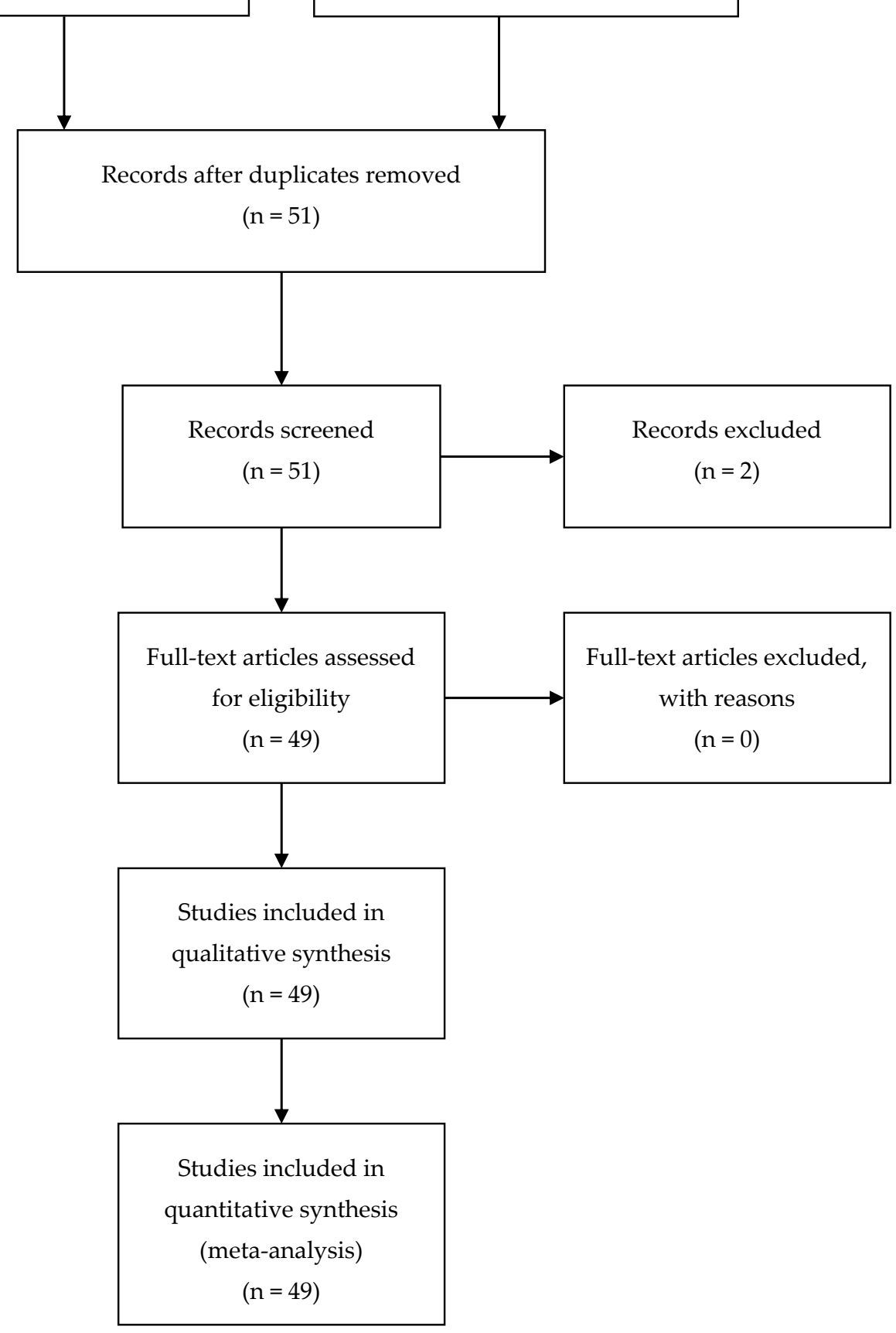

Figure A1. PRISMA Flow diagram. From: [74]. 


\section{References}

1. Nakamoto, S. Bitcoin: A Peer-to-Peer Electronic Cash System. 2008. Available online: https://bitcoin.org/ bitcoin.pdf (accessed on 25 February 2019).

2. Tschorsch, F.; Scheuermann, B. Bitcoin and beyond: A technical survey on decentralized digital currencies. IEEE Commun. Surv. Tutor. 2015, 18, 2084-2123. [CrossRef]

3. Diffie, W.; Hellman, M. New directions in cryptography. IEEE Trans. Inf. Theory 1976, 22, 644-654. [CrossRef]

4. Konashevych, O.; Poblet, M. Is Blockchain Hashing an Effective Method for Electronic Governance? arXiv 2018, arXiv:1810.08783.

5. Macrinici, D.; Cartofeanu, C.; Gao, S. Smart contract applications within blockchain technology: A systematic mapping study. Telemat. Inform. 2018, 35, 2337-2354. [CrossRef]

6. Eyal, I.; Gencer, A.E.; Sirer, E.G.; Van Renesse, R. Bitcoin-NG: A Scalable Blockchain Protocol. In Proceedings of the 13th USENIX Symposium on Networked Systems Design and Implementation, Santa Clara, CA, USA, 16-18 March 2016; pp. 45-59.

7. Fanning, K.; Centers, D.P. Blockchain and Its Coming Impact on Financial Services. J. Corp. Account. Financ. 2016, 27, 53-57. [CrossRef]

8. Kiayias, A.; Lamprou, N.; Stouka, A.-P. Proofs of Proofs of Work with Sublinear Complexity. In International Conference on Financial Cryptography and Data Security; Springer: Berlin/Heidelberg, Germany, 2016; Volume 9604, pp. 61-78.

9. Mills, D.; Wang, K.; Malone, B.; Ravi, A.; Marquardt, J.; Chen, C.; Badev, A.; Brezinski, T.; Fahy, L.; Liao, K.; et al. Distributed Ledger Technology in Payments, Clearing, and Settlement. Financ. Econ. Discuss. Ser. 2016, 2016. [CrossRef]

10. Hochstein, M. Fintech (the word, that is) Evolves. American Banker. 2015. Available online: https: //www.americanbanker.com/opinion/FinTech-the-word-that-is-evolves (accessed on 23 June 2019).

11. Arner, D.W.; Barberis, J.; Buckley, R.P. 150 Years of FinTech: An evolutionary analysis. Finsia J. Appl. Financ. 2016, 3, 22-29.

12. Du, W.; Pan, S.L.; Leidner, D.E.; Ying, W. Affordances, experimentation and actualization of FinTech: A blockchain implementation study. J. Strateg. Inf. Syst. 2019, 28, 50-65. [CrossRef]

13. Ramanathan, R.; Ramanathan, U.; Bentley, Y. The debate on flexibility of environmental regulations, innovation capabilities and financial performance-A novel use of DEA. Omega 2018, 75, 131-138. [CrossRef]

14. Szabo, N. Smart Contracts. Unpublished work. 1994.

15. Natoli, C.; Gramoli, V. The Blockchain Anomaly. In Proceedings of the IEEE 15th International Symposium on Network Computing and Applications (NCA), Cambridge, MA, USA, 31 October-2 November 2016; pp. 310-317.

16. Gu, Y.; Hou, D.D.; Wu, X.H.; Tao, J.; Zhang, Y.Q. Decentralized Transaction Mechanism Based on Smart Contract in Distributed Data Storage. Information 2018, 9, 286. [CrossRef]

17. Atzei, N.; Bartoletti, M.; Cimoli, T. A Survey of Attacks on Ethereum Smart Contracts (SoK); Springer Science and Business Media LLC: Berlin/Heidelberg, Germany, 2017; Volume 10204, pp. 164-186.

18. Petersen, K.; Feldt, R.; Mujtaba, S.; Mattsson, M. Systematic Mapping Studies in Software Engineering. In Proceedings of the 12th International Conference on Evaluation and Assessment in Software Engineering, EASE'08, Bari, Italy, 26-27 June 2008; pp. 68-77.

19. Kitchenham, B.; Brereton, O.P.; Budgen, D.; Turner, M.; Bailey, J.; Linkman, S. Systematic literature reviews in software engineering - A systematic literature review. Inf. Softw. Technol. 2009, 51, 7-15. [CrossRef]

20. Bharti, K.; Agrawal, R.; Sharma, V. Value co-creation: Literature review and proposed conceptual framework. Int. J. Mark. Res. 2015, 57, 571. [CrossRef]

21. Centobelli, P.; Cerchione, R.; Esposito, E. Shashi Exploration and exploitation in the development of more entrepreneurial universities: A twisting learning path model of ambidexterity. Technol. Forecast. Soc. Chang. 2019, 141, 172-194. [CrossRef]

22. Dybå, T.; Dingsøyr, T. Empirical studies of agile software development: A systematic review. Inf. Softw. Technol. 2008, 50, 833-859. [CrossRef]

23. Matsuura, K. Token Model and Interpretation Function for Blockchain-Based FinTech Applications. IEICE T FUND ELECTR 2019, E102.A, 3-10. [CrossRef] 
24. Sawa, T. Blockchain technology outline and its application to field of power and energy system. Electr. Eng. Jpn. 2019, 206, 11-15. [CrossRef]

25. Almeida, S.; Albuquerque, A.; Silva, A. An Approach to Develop Software that Uses Blockchain. Softw. Eng. Algorithms Intell. Syst. 2019, 763, 346-355. [CrossRef]

26. Cai, C.W. Disruption of financial intermediation by FinTech: A review on crowdfunding and blockchain. Account. Financ. 2018, 58, 965-992. [CrossRef]

27. Adhami, S.; Giudici, G.; Martinazzi, S. Why do businesses go crypto? An empirical analysis of initial coin offerings. J. Econ. Bus. 2018, 100, 64-75. [CrossRef]

28. Kumar, S.; Mookerjee, V.; Shubham, A. Research in Operations Management and Information Systems Interface. Prod. Oper. Manag. 2018, 27, 1893-1905. [CrossRef]

29. Dimbean-Creta, O. Fintech in Corporations. Transforming the Finance Function. Qual. Access Success 2018, 19, 21-28.

30. Dimbean-Creta, O. Fintech-Already New Fashion in Finance, but what about the Future? Qual. Access Success 2017, 18, 25-29.

31. Deng, H.; Huang, R.H.; Wu, Q. The Regulation of Initial Coin Offerings in China: Problems, Prognoses and Prospects. Eur. Bus. Organ. Law Rev. 2018, 19, 465-502. [CrossRef]

32. Ashta, A.; Biot-Paquerot, G. FinTech evolution: Strategic value management issues in a fast changing industry. Strat. Chang. 2018, 27, 301-311. [CrossRef]

33. Tseng, J.-H.; Liao, Y.-C.; Chong, B.; Liao, S.-W. Governance on the Drug Supply Chain via Gcoin Blockchain. Int. J. Environ. Res. Public Health 2018, 15, 1055. [CrossRef]

34. Stefan, C. Tales from the crypt: Might cryptocurrencies spell the death of traditional money? A quantitative analysis. In Proceedings of the International Conference on Business Excellence, Bucharest, Romania, 15 Jun 2018; Volume 12, pp. 918-930.

35. Marsal-Llacuna, M.-L. Future living framework: Is blockchain the next enabling network? Technol. Forecast. Soc. Chang. 2018, 128, 226-234. [CrossRef]

36. Liang, J.; Li, L.; Zeng, D.; Zhao, Y. Correlation-based Dynamics and Systemic Risk Measures in the Cryptocurrency Market. In Proceedings of the IEEE International Conference on Intelligence and Security Informatics (ISI), Miami, FL, USA, 8-10 November 2018; pp. 43-48.

37. Kim, S.; Sarin, A. Distributed Ledger and Blockchain Technology: Framework and Use Cases. SSRN Electron. J. 2018, 16, 90-101. [CrossRef]

38. Rodrigues, B.; Bocek, T.; Stiller, B. The Use of Blockchains: Application-Driven Analysis of Applicability. Blockchain Technol. 2018, 111, 163-198. [CrossRef]

39. Chuen, D.L.K.; Deng, R. Handbook of Blockchain, Digital Finance, and Inclusion: Cryptocurrency, FinTech, InsurTech, and Regulation Preface, 1st ed.; Academic Press Ltd.: Cambridge, MA, USA; Elsevier Science Ltd.: Amsterdam, The Netherlands, 2017; Volume 1.

40. Raikwar, M.; Mazumdar, S.; Ruj, S.; Gupta, S.S.; Chattopadhyay, A.; Lam, K.-Y. A Blockchain Framework for Insurance Processes. In Proceedings of the 9th IFIP International Conference on New Technologies, Mobility and Security (NTMS), Paris, France, 26-28 February 2018; pp. 1-4.

41. Irwin, A.S.; Turner, A.B. Illicit Bitcoin transactions: Challenges in getting to the who, what, when and where. J. Money Laund. Control 2018, 21, 297-313. [CrossRef]

42. Allon, F. Money after Blockchain: Gold, Decentralised Politics and the New Libertarianism. Aust. Fem. Stud. 2018, 33, 223-243. [CrossRef]

43. Karkkainen, T.; Panos, G.A.; Broby, D.; Bracciali, A. On the Educational Curriculum in Finance and Technology. In International Conference on Internet Science; Springer: Berlin/Heidelberg, Germany, 2018; Volume 10750, pp. 7-20.

44. Yang, D.; Li, M. Evolutionary Approaches and the Construction of Technology-Driven Regulations. Emerg. Mark. Financ. Trade 2018, 54, 3256-3271. [CrossRef]

45. Shkodina, I.V.; Timoshenkov, I.V.; Nashchekina, O.N. The impact of financial technology on the transformation of the financial system. Financ. Crédit. Act. Probl. Theory Pract. 2018, 1, 417-424. [CrossRef]

46. Yavuz, E.; Koc, A.K.; Cabuk, U.C.; Dalkilic, G. Towards secure e-voting using ethereum blockchain. In Proceedings of the 6th International Symposium on Digital Forensic and Security (ISDFS), Antalya, Turkey, 22-25 March 2018; pp. 1-7. 
47. Gomber, P.; Kauffman, R.J.; Parker, C.; Weber, B.W. On the Fintech Revolution: Interpreting the Forces of Innovation, Disruption, and Transformation in Financial Services. J. Manag. Inf. Syst. 2018, 35, 220-265. [CrossRef]

48. Olsen, R.; Battiston, S.; Caldarelli, G.; Golub, A.; Nikulin, M.; Ivliev, S.; Buchanan, B.; Tasca, P. Case study of Lykke exchange: Architecture and outlook. J. Risk Financ. 2018, 19, 26-38. [CrossRef]

49. Kavassalis, P.; Stieber, H.; Breymann, W.; Saxton, K.; Gross, F.J.; Buchanan, B.; Tasca, P. An innovative RegTech approach to financial risk monitoring and supervisory reporting. J. Risk Financ. 2018, 19, 39-55. [CrossRef]

50. Zalan, T. Born global on blockchain. Rev. Int. Bus. Strat. 2018, 28, 19-34. [CrossRef]

51. Ducas, E.; Wilner, A. The security and financial implications of blockchain technologies: Regulating emerging technologies in Canada. Int. J. Can. J. Glob. Policy Anal. 2017, 72, 538-562. [CrossRef]

52. Chen, Z.; Li, Y.; Wu, Y.; Luo, J. The transition from traditional banking to mobile internet finance: An organizational innovation perspective-A comparative study of Citibank and ICBC. Financ. Innov. 2017, 3, 12. [CrossRef]

53. Larios-Hernández, G.J. Blockchain entrepreneurship opportunity in the practices of the unbanked. Bus. Horizons 2017, 60, 865-874. [CrossRef]

54. Del Rio, C.A. Use of distributed ledger technology by central banks: A review. Enfoque Ute 2017, 8, 1-13. [CrossRef]

55. Eyal, I. Blockchain Technology: Transforming Libertarian Cryptocurrency Dreams to Finance and Banking Realities. Computer 2017, 50, 38-49. [CrossRef]

56. Park, J.H.; Park, J.H. Blockchain Security in Cloud Computing: Use Cases, Challenges, and Solutions. Symmetry 2017, 9, 164. [CrossRef]

57. Alabi, K. Digital blockchain networks appear to be following Metcalfe's Law. Electron. Commer. Res. Appl. 2017, 24, 23-29. [CrossRef]

58. Marsal-Llacuna, M.-L.; Oliver-Riera, M. The standards revolution: Who will first put this new kid on the blockchain? In Proceedings of the ITU Kaleidoscope: Challenges for a Data-Driven Society (ITU K), Nanjing, China, 27-29 November 2017.

59. Kostin, G.A.; Pokrovskaia, N.N.; Ababkova, M.U. Master-chain as an intellectual governing system for producing and transfer of knowledge. In Proceedings of the IEEE II International Conference on Control in Technical Systems (CTS), St. Petersburg, Russia, 25-27 October 2017; pp. 71-74.

60. Nakashima, H.; Aoyama, M. An Automation Method of SLA Contract of Web APIs and Its Platform Based on Blockchain Concept. In Proceedings of the IEEE International Conference on Cognitive Computing (ICCC), Honolulu, HI, USA, 25-30 June 2017; pp. 32-39.

61. Mannaro, K.; Pinna, A.; Marchesi, M. Crypto-trading: Blockchain-oriented energy market. In Proceedings of the AEIT International Annual Conference, Cagliari, Italy, 20-22 September 2017.

62. Zook, M.A.; Blankenship, J. New spaces of disruption? The failures of Bitcoin and the rhetorical power of algorithmic governance. Geoforum 2018, 96, 248-255. [CrossRef]

63. Chen, P.-W.; Jiang, B.-S.; Wang, C.-H. Blockchain-based payment collection supervision system using pervasive Bitcoin digital wallet. In Proceedings of the IEEE 13th International Conference on Wireless and Mobile Computing, Networking and Communications (WiMob), Rome, Italy, 9-11 October 2017; pp. 139-146.

64. Cachin, C. Blockchains and Consensus Protocols: Snake Oil Warning. In Proceedings of the 13th European Dependable Computing Conference (EDCC), Geneva, Switzerland, 4-8 September 2017; pp. 1-2.

65. Zavolokina, L.; Dolata, M.; Schwabe, G. FinTech Transformation: How IT-Enabled Innovations Shape the Financial Sector. In Exploring Service Science; Springer Science and Business Media LLC: Berlin/Heidelberg, Germany, 2017; Volume 276, pp. 75-88.

66. Nor, R.M.; Rahman, M.M.H.; Rahman, T.; Abdullah, A. Blockchain Sadaqa Mechanism for Disaster Aid Crowd Funding. In Proceedings of the 6th International Conference on Computing and Informatics: Embracing Eco-Friendly Computing, Kuala Lumpur, 25-27 April 2017; pp. 400-405.

67. Zhao, H.; Zhang, Y.; Peng, Y.; Xu, R. Lightweight Backup and Efficient Recovery Scheme for Health Blockchain Keys. In Proceedings of the IEEE 13th International Symposium on Autonomous Decentralized System (ISADS), Bangkok, Thailand, 22-24 March 2017; pp. 229-234.

68. Yamasaki, S.; Okada, H.; Bracamonte, V. Proposed classification of blockchains based on authority and incentive dimensions. In Proceedings of the 19th International Conference on Advanced Communication Technology (ICACT), PyeongChang, Korea, 19-22 February 2017; pp. 593-597. 
69. Wu, S.; Guo, D. Research into Information Security Strategy Practices for Commercial Banks in Taiwan. Recent Dev. Intell. Syst. Interact. Appl. 2017, 541, 182-187. [CrossRef]

70. Tasca, P.; Aste, T.; Pelizzon, L.; Perony, N. Banking Beyond Banks and Money-A Guide to Banking Services in the Twenty-First Century Introduction; Springer: New York, NY, USA, 2016. [CrossRef]

71. Treiblmaier, H.; Beck, R. Business Transformation through Blockchain; Palgrave Macmillan: Cham, Switzerland, 2019; Volume 1. [CrossRef]

72. Fernández, A.; Insfrán, E.; Abrahão, S. Usability evaluation methods for the web: A systematic mapping study. Inf. Softw. Technol. 2011, 53, 789-817. [CrossRef]

73. Centobelli, P.; Cerchione, R.; Esposito, E. Knowledge Management in Startups: Systematic Literature Review and Future Research Agenda. Sustainability 2017, 9, 361. [CrossRef]

74. Moher, D.; Liberati, A.; Tetzlaff, J.; Altman, D.G. Preferred Reporting Items for Systematic Reviews and Meta-Analyses: The PRISMA Statement. Ann. Intern. Med 2009, 151, 264-269. [CrossRef]

(C) 2019 by the authors. Licensee MDPI, Basel, Switzerland. This article is an open access article distributed under the terms and conditions of the Creative Commons Attribution (CC BY) license (http://creativecommons.org/licenses/by/4.0/). 